\title{
ArcheoSciences
}

Revue d'archéométrie

\section{Nouvelles recettes et vieilles marmites. Quelques regards sur les analyses des matériaux minéraux utilisés par l'homme dans le passé}

New recipes and old pots. Some glances on the analyses of mineral material used by Man in the Past

\section{Vincent Serneels}

\section{OpenEdition \\ Journals}

\section{Édition électronique}

URL : https://journals.openedition.org/archeosciences/5479

DOI : 10.4000/archeosciences.5479

ISSN : 2104-3728

\section{Éditeur}

Presses universitaires de Rennes

\section{Édition imprimée}

Date de publication : 27 juin 2018

Pagination : 109-112

ISBN : 978-2-7535-7587-5

ISSN : 1960-1360

\section{Référence électronique}

Vincent Serneels, « Nouvelles recettes et vieilles marmites. Quelques regards sur les analyses des matériaux minéraux utilisés par l'homme dans le passé », ArcheoSciences [En ligne], 42-1 | 2018, mis en ligne le 27 juin 2020, consulté le 28 janvier 2022. URL : http://journals.openedition.org/ archeosciences/5479; DOI : https://doi.org/10.4000/archeosciences.5479 


\title{
Nouvelles recettes et vieilles marmites. Quelques regards sur les analyses des matériaux minéraux utilisés par l'homme dans le passé
}

\author{
New Recipes and Old Pots. Some Glances on the Analyses \\ of Mineral Material Used by Man in the Past
}

\author{
Vincent SERnEELS ${ }^{a}$
}

\begin{abstract}
Résumé : Les matériaux minéraux résistent bien à l'enfouissement et forment donc une large part de l'enregistrement archéologique. La caractérisation de ces matériaux à l'aide des méthodes des sciences naturelles contribue significativement à la compréhension des sociétés anciennes à travers l'identification des techniques et des échanges.

Au cours des 40 dernières années, la gamme des matériaux étudiés et les problématiques de base n’ont pratiquement pas changé. Par contre, les moyens d'analyse ont été perfectionnés de manière cruciale permettant une augmentation extraordinaire de la qualité et la quantité des données. Dans bien des domaines, l'avancée des connaissances a donc été spectaculaire permettant de renouveler complètement notre compréhension de l'histoire des techniques et de l'économie.
\end{abstract}

Abstract: The mineral materials are quite resistant to burial conditions and form a major part of the archaeological record. The characterization of these materials using the Natural Sciences methods contributes significantly to the understanding of the past societies through the identification of technologies and exchange networks.

During the last 40 years, the range of the analysed materials and the basic problematics did not change virtually. On the contrary, the means of analysis improved drastically allowing an extraordinary increase of the quality and quantity of data. In many cases, the improvement of knowledge has been spectacular inducing the complete renewal of our understanding of the history of technology and economy.

Mots clés : Provenance, Technologie, Roche, Céramique, Verre, Métaux.

Keywords: Provenance, Technology, Stone, Ceramic, Glass, Metals.

\section{LeS MATÉRIAUX MiNÉRAUX}

Les matériaux minéraux au sens large, occupent une place de choix parmi les vestiges matériels de l'activité humaine. Aux origines, certaines roches naturelles, comme les silex, permettent à l'homme de façonner des armes et des outils indispensables. Avec la maitrise de la cuisson de la céramique, il devient possible de produire des récipients efficaces. Les métaux vont progressivement révolutionner les techniques et les sociétés. Pour la construction des bâtiments, pierres et mortiers sont des ressources primordiales. D'autres matériaux s'ajoutent à la liste, comme les verres, de nombreux pigments, les pierres précieuses, le sel, les aluns, etc. Dans certains cas, ce sont des matériaux naturels non transformés, mais le plus souvent, ce sont des matériaux artificiels fabriqués par l'homme.

a Professeur de pétrologie appliquée, Département de Géosciences, Université de Fribourg, Chemin du Musée 6, 1700 FRIBOURG, Suisse. (vincent.serneels@ unifr.ch) 
À la base de toutes les recherches dans le domaine, on retrouve une démarche de caractérisation des matériaux étudiés qui s’appuie généralement sur la description des propriétés macroscopiques (couleur, densité, etc.), la composition chimique élémentaire et/ou isotopique, l'identification des constituants minéraux et autres ainsi que l'étude de la texture (taille et morphologie des constituants, proportions et disposition spatiale des constituants). Cette démarche fait toujours appel à des observations à différentes échelles. L'étude des surfaces (corrosion, traces de façonnage et d'utilisation) contribue aussi significativement à la compréhension des objets et de leur utilisation. Dans le cas idéal, une caractérisation devrait être complète. Dans la pratique, d'une part, il faut souvent renoncer à certaines approches afin de ne pas mettre en danger l'intégrité de l'objet et d'autre part, les moyens disponibles pour l'étude sont limités et imposent des choix méthodologiques.

Au-delà de la caractérisation, les études de provenance visent à établir un lien entre un objet et le lieu de l'extraction de la matière première. Ces liens sont généralement interprétés en termes d'échanges économiques. Ce sont des démarches comparatives qui demandent une connaissance approfondie des ressources potentielles (banques de données de référence) et un traitement statistique de l'information. Dans le cas des matériaux transformés, il est indispensable de comprendre aussi les processus de production (chaînes opératoires techniques) pour identifier des paramètres qui permettent d'établir une filiation entre l'objet et le gisement naturel.

Les études sur les technologies ont pour but de comprendre les processus de fabrication des objets. Comme le matériau tend à enregistrer principalement la dernière étape de la transformation, l'approche doit être complétée par l'étude des installations et des déchets de production. Les approches expérimentales et ethnoarchéologiques enrichissent considérablement la réflexion.

\section{LE DÉVELOPPEMENT DES MOYENS D’ANALYSE}

Après l'âge des pionniers qui, au XIX ${ }^{\mathrm{e}}$ siècle déjà, furent les premiers à analyser des objets archéologiques du point de vue des sciences des matériaux, plusieurs techniques de base vont se développer : les techniques traditionnelles de la chimie par voie humide, la diffraction des rayons $\mathrm{X}$ et la microscopie optique.

À partir des années 1960, l'analyse des matériaux se renforce considérablement grâce au développement des méthodes de spectrométrie basées sur les interactions entre les rayonnements électromagnétiques et la matière (UV-V, IR, Raman, OES, AAS, XRF, NAA, etc.). Dans les années
1970, des appareils fiables sont progressivement mis sur le marché et trouvent leurs places dans les universités et les organismes de recherche. Ils restent délicats à utiliser mais ils permettent une augmentation très significative du nombre d'analyse. Progressivement, l'informatique et les ordinateurs renforcent l'efficacité des appareils et simplifient leur utilisation. Certains chercheurs se spécialisent dans le domaine, se regroupent et forment des équipes qui ont parfois leurs propres équipements lourds. La discipline se structure avec la mise en place de réseaux nationaux et internationaux, en Europe et aux États-Unis et les premières revues scientifiques (Archaeometry, 1958; Journal of Archaeological Sciences, 1974; Revue d'Archéométrie, 1977).

À partir de la décennie 1980, il devient possible de réaliser des analyses spectrométriques presque en routine. Une nouvelle approche se développe, celle de la spectrométrie de masse (MS). La matière est ionisée puis les ions sont séparés en fonction du rapport masse/charge. Dix ans plus tard, grâce aux torches à plasma on obtient une ionisation efficace (ICP-MS). Au début des années 2000, ces systèmes sont couplés avec un dispositif d'ablation laser (LA-ICP-MS). En parallèle, on assiste au développement de la micro-analyse, grâce au microscope électronique à balayage (MEB) puis à la microsonde électronique (EPMA). Dans les années 2000, une nouvelle étape commence avec les synchrotrons.

\section{RÉFLEXIONS SUR LES ÉVOLUTIONS DANS LA RECHERCHE SUR LES MATÉRIAUX MINÉRAUX UTILISÉS PAR L'HOMME AU COURS DES 40 DERNIÈRES ANNÉES (1977-2017)}

Au cours des 40 dernières années, l'augmentation des possibilités analytiques a été spectaculaire. Cette augmentation concerne la diversité des méthodes et la précision des mesures. Elle se marque aussi dans les quantités toujours plus grandes des données analytiques, à la fois parce que le nombre de machines augmente et que le temps d'acquisition diminue. Enfin, il y a une autre évolution essentielle vers la miniaturisation et la portabilité des appareillages.

Toutes ces évolutions marquent la pratique dans le domaine de la recherche sur les matériaux minéraux utilisés par l'homme. Dès que les méthodes d'analyse dépassent l'étape de la mise au point, elles sont presque immédiatement appliquées à des matériaux anciens. Quand des appareillages fiables et relativement simples permettent d'obtenir des analyses de routine, elles sont mises en œuvre pour aborder les problématiques qui demandent des séries d'analyses quantitativement importantes comme les études de provenance. À cause de la nature même des objets archéo- 
logiques, par définition irremplaçables, il y a une certaine pression pour développer des méthodes non destructrices ou au moins peu invasives. Les appareillages portables ouvrent de nouvelles perspectives pour les études in situ des objets intransportables ou difficilement transportables.

Par contre, la gamme des matériaux qui sont étudiés n’a pas beaucoup changé. Il y a 40 ans, les chercheurs analysaient déjà des roches, des céramiques, des verres, des métaux, des pigments et des déchets pyrotechnologiques. Les problématiques fondamentales qui, au-delà de la caractérisation des matériaux vont vers les études technologiques et les études de provenance, sont restées presque inchangées dans leurs grandes lignes. On constate cependant que les travaux récents montrent une forte tendance à devenir plus globalisants et pluridisciplinaires. Les études portant sur un objet ou une classe d'objets et mettant en œuvre une seule méthode existent toujours. Cependant, la tendance est à la mise en œuvre conjointe de méthodes complémentaires pour appréhender le matériau ou l'objet de manière globale et multiscalaire. Dans certains cas, ces études peuvent être extrêmement sophistiquées et jouent un rôle très important d'incubateur méthodologique autour d'un défi analytique particulièrement complexe.

Les études sur des objets complexes, comme un monument, un site, un paysage, ou sur des systèmes complexes, comme une chaîne opératoire ou un système d'échange, sont de plus en plus fréquentes. Elles sont forcément multiméthodes et pluridisciplinaires.

Ce qui a fondamentalement changé dans la pratique de ces recherches, c'est qu'une incroyable masse de connaissances nouvelles a été acquise en 40 ans. Pour la plupart des matériaux, dans un grand nombre de domaines, des travaux de longue haleine ont été réalisés et bien souvent, ils ont abouti à une modification complète des paradigmes précédents. Ce sont souvent des recherches qui s'étalent sur plusieurs décennies et qui sont des œuvres de recherche collectives. Ce sont de véritables réseaux de chercheurs qui se sont réunis autour d'un thème et qui, à travers l'entraide et la compétition scientifique ont ainsi fait bouger notre compréhension de grandes questions.

Les exemples sont nombreux et on ne peut prétendre à dresser une liste exhaustive ni à établir un classement de prestige. Il est tout de même utile de citer quelques exemples. Pour le cuivre, des progrès fantastiques ont été accomplis, en particulier pour les périodes les plus anciennes, dans la compréhension de l'extraction minière, de la production du métal et des techniques. En Méditerranée orientale et dans les Balkans, les études de provenance permettent de décrire la circulation du métal à l'Âge du Bronze. Pour l'or, il reste difficile d'établir une traçabilité mais les analyses de composition permettent une réflexion renouvelée sur l'or monnayé et son altération. Pour tous les métaux, mais surtout l'argent, les études sur les isotopes du plomb se sont révélées complexes mais puissantes. La chaîne opératoire de la métallurgie du fer a été mise en évidence. Les recherches récentes ouvrent la voie aux études de provenance pour ce métal. Pour les verres, les analyses chimiques jouent un rôle prépondérant dans la mise en évidence de la production de masse du verre brut au Levant pendant la période romaine. L'évolution technologique entre les verres de l'Âge du Bronze et ceux du Moyen Âge peut maintenant être retracée. Les grandes carrières de marbres blancs et colorés de l'Antiquité sont identifiées et caractérisées. Les sources d'obsidienne en Méditerranée sont très bien connues, mais il reste du travail sur celles d'Anatolie et du Caucase. Les techniques des céramiques fines rouges et noires de l'Antiquité ont fait l'objet de travaux approfondis. Les études de provenance sur les amphores éclairent les échanges commerciaux de l'Empire Romain. Les technologies sophistiquées des céramiques islamiques, comme le lustre métallique, ont été étudiées en détail.

Incontestablement, la recherche sur les matériaux minéraux a marqué des progrès essentiels au cours des 40 dernières années. Les acquis sont considérables mais il n'en reste pas moins d'importantes lacunes. En particulier, il y a des déséquilibres très importants dans la recherche. On constate avant tout que les périodes anciennes sont nettement favorisées au détriment des plus récentes. En particulier, les études portant sur le Moyen Âge et les Temps Modernes sont encore très lacunaires. Il y a aussi un fort déséquilibre en faveur des matériaux précieux et des objets prestigieux. Ce n'est pas pour surprendre dans la mesure où ce sont des tendances de l'archéologie elle-même. Un autre aspect est le déséquilibre en faveur des pays développés. De manière presque exclusive, c'est dans ces pays que se pratique l'archéométrie, ce qui est logique dans la mesure où cette discipline demande des moyens financiers, des installations coûteuses et des personnels hautement qualifiés. 


\section{Bibliographie}

C'est à travers les actes des colloques spécialisés que l'on peut appréhender au mieux l'évolution de la recherche sur les différents groupes de matériaux minéraux. Avec par exemple les conferences suivantes :

- pour les roches : ASMOSIA Association for the Study of Marble and Other Stones In Antiquity,

- pour les céramiques : EMAC European Meeting on Ancient Ceramic,

- pour les verres : AIHV Association pour l'Histoire du Verre,

- pour les métaux : Archaeometallurgy in Europe.

Références des derniers actes publiés suite à ces conférences :

Guitierrez Garcia M. A., Lapuente P., Roda I. (éd.), 2012. Interdisciplinary Studies on Ancient Stones: Proceedings of the
IX ASMOSIA Conference, Tarragona, Spain, June 8-13, 2009, Documenta 23, Institut Catala d'Arqueologia Classica, Tarragona.

Maritan L., Grifa C., Nodari L. (éd.), 2013. EMAC 2013, Inside the pottery: composition, tchnology, sources, provenance and use, 19-21 September 2013 Padova, Periodico di Mineralogia 84/1 (Special Issue).

Wolf S., De Pury-Gysel A. (éd.), 2017. Annales du $20^{\circ}$ Congrès de l'Association Internationale pour l'Histoire du Verre, Fribourg, Romont 7-11 septembre 2015, Verlag Marie Leidorf GmbH, Rahden.

Hauptmann A., Modarressi-Tehrani D. (éd.), 2015. Archaeometallurgy in Europe III, Proceedings of the $3^{\text {rd }}$ International Conference Deutsches Bergbau Museum Bochum, June 29-July 1, 2011, Der Anschnitt, Zeitschrift für Kunst und Kultur im Bergabau, Beiheft 26, Bochum. 